\title{
Glioma localization and excision using direct electrical stimulation for language mapping during awake surgery
}

\author{
TIANDONG LI, HONGMIN BAI, GUOLIANG WANG, WEIMIN WANG, JIAN LIN, \\ HAN GAO, LIMIN WANG, LIHUI XIA and XUEMIN XIE
}

Department of Neurosurgery, Liuhuaqiao Hospital, Guangzhou, Guangdong 510010, P.R. China

Received June 3, 2014; Accepted February 4, 2015

DOI: $10.3892 /$ etm.2015.2359

\begin{abstract}
The aim of this study was to investigate the method and significance of the application of direct electrical stimulation (DES) to the brain mapping of language functions during glioma surgery. A retrospective analysis of clinical data was performed for 91 cases of brain functional area glioma surgery under DES from January 2003 until January 2012. Following cortical electrical stimulation, 88 patients exhibited seizures involving facial or hand movements and 91 cases experienced language disorders such as counting interruption, naming errors or anomia. The most commonly observed areas of counting interruption were distributed on the posterior part of the left anterior central gyrus $(47.7 \%)$, the operculum of the left inferior frontal gyrus $(24.4 \%)$ and the triangular part of the left inferior frontal gyrus (12.8\%). Postoperative magnetic resonance imaging demonstrated that overall excision was achieved in 53 cases and sub-overall excision was performed in 31 cases. A total of 42 cases $(46.2 \%)$ exhibited no postoperative neurological dysfunction, 39 cases (42.9\%) exhibited brief language dysfunction, 27 cases (29.7\%) experienced brief limb movement disorder, and one case appeared to have permanent neurological dysfunction. DES was indicated to be a reliable and noninvasive method for the intraoperative positioning of language areas, and was able to resect gliomas in the language area with maximal safety.
\end{abstract}

\section{Introduction}

The ideal goal of brain tumor surgery treatment is to maximize the resection of tumor volume, without damaging motion, sensation, language and other important cognitive functions. The key in this type of surgery is how to identify the positions of brain areas associated with important functions during the

Correspondence to: Professor Weimin Wang, Department of Neurosurgery, Liuhuaqiao Hospital, 111 Liuhua Road, Guangzhou, Guangdong 510010, P.R. China

E-mail: weiminwangdoc@126.com

Key words: glioma, cerebral localization, direct electrical stimulation, awake craniotomy surgery accurately and in real time. Accurate positioning may avoid excessive resection-induced permanent neurological dysfunction, and also avoid incomplete excision due to excessive carefulness, in which situation the desired therapeutic effect would be challenging to achieve (1-3). In the positioning of cerebral primary functions such as motion and sensation, preoperative positioning with magnetic resonance imaging (MRI) or intraoperative direct electrical stimulation (DES) positioning is currently well developed. However, clinical problems remain in the positioning of higher cognitive functions, such as language and memory.

Language function is a human-specific high-level cognitive function; in comparison with motion, sensation and vision, for example, the language-related cortex is distributed more widely and in a much more complex manner inside the human brain. The cortical localization of language varies in different individuals, particularly in patients with intracranial lesions where the atypical distribution of the language cortex is particularly common (4). The ability to locate the language cortex is the key towards surgery in the dominant hemisphere, particularly surgery of lesions close to the language area. How best to perform resection of lesions in the language area of the brain without inducing a postoperative language disorder, thus protecting the patient's quality of life, has become an issue of particular concern in neurosurgery currently (5-8).

As the most accurate and reliable method of brain functional area positioning, DES is able to determine in real time the parts of the brain necessary for such functions as movement, sensation, language, and even memory. A recent meta-analysis suggested that it could also improve the degree of resection of glioma while reducing the incidence of permanent neurological dysfunction (9).

Investigations of the distribution characteristics of Chinese language function in the cortex are limited to the application of functional magnetic resonance in China. Chinese and English are significantly different languages. Chinese involves the characteristics of shape, meaning and grammar, and learning it is a comprehensive study involving calligraphy and pronunciation. English, however, involves phonetic learning, and a focus on speaking. Thus, there may be some differences in the language areas between Chineseand English-speaking populations. Tan et al of Hong Kong University found that native Chinese speakers and native English speakers exhibited differences in anatomical struc- 
tures; the left inferior frontal gyrus, middle posterior frontal gyrus and left anterocentral temporal lobe of native Chinese speakers were relatively larger than those of native English speakers (10).

The present authors have carried out resections of functional area lesions in the awake status of general anesthesia in China since 2003, with the use of intraoperative DES to position the functional area cortex $(11,12)$. The present study summarizes the clinical experience of 91 cases, in which intraoperative DES was applied during the resection of glioma in the brain language area, aiming to summarize distribution rules for the Chinese language cortex and the significance of DES in the resection of gliomas in the language area.

\section{Materials and methods}

Study eligibility. Patients were considered eligible for this prospective longitudinal study (conducted from January 2003 to January 2012) if they: i) had suspected neuroepithelial tumors near or within eloquent areas; ii) were between 16 and 60 years of age; and iii) were fluent in speaking and understanding the Chinese language. Patients were excluded from the study if they had severe preoperative deficits or were mentally disoriented. This study was conducted in accordance with the Declaration of Helsinki. The study procedure was approved by the Medical Ethics Committee of Liuhuaqiao Hospital (Guangzhou, China). Written informed consent was obtained from all patients.

Preoperative evaluation. Detailed neurological and psychological assessments were performed for all patients. General cognitive function was assessed used the mini-mental state examination (MMSE). The evaluation of handedness judgment was conducted using the Edinburgh handedness examination (13). Three days prior to surgery, the psychiatrist responsible for intraoperative monitoring explained the surgery to the patient, and screened the intraoperative tasks. Conventional MRI plain and enhanced scans were performed preoperatively, and patients admitted after 2006 were examined by functional MRI examination or diffusion tensor imaging (DTI), magnetic resonance spectroscopy (MRS) and blood oxygen level dependent-functional magnetic resonance imaging (BOLD-fMRI).

\section{Surgical treatment}

Anesthesia. The intraoperative awake method of general anesthesia was used (3). Intubation was performed through a laryngeal mask, and the anesthesia used was propofol for general anesthesia, bupivacaine or ropivacaine for local anesthesia of the scalp (including the supraorbital nerve, auriculotemporal nerve, greater occipital nerve and ear nervelet, as well as infiltration anesthesia around incision area) and lidocaine for local anesthesia of the meninges. Following opening of the meninges, the propofol administration was stopped, the patient was woken up and the laryngeal mask was unplugged. Following tumor removal, the patient was anesthetized again and the laryngeal mask was reapplied.

Anatomical localization of lesions. Intraoperative B-ultrasound and/or MRI neuronavigation was performed to determine the location and extent of the lesions.
Brain area DES method. The DES method was conducted as previously described (11). Bipolar electrical nerve stimulation (interval, $5 \mathrm{~mm}$; OSIRIS NeuroStimulator; Inomed Medizintechnik GmbH, Teningen, Germany) was used. The stimulation range covered all areas of the exposed cortex and suspicious subcortical regions; the frequency was $60 \mathrm{~Hz}$, the pulse duration was $1 \mathrm{msec}$, and the current was $0.5-10 \mathrm{~mA}$ (usually 4-6 mA). The stimulus duration of every point was $1 \mathrm{sec}$ for motion and sensation tasks and $4 \mathrm{sec}$ for language tasks.

Observation indices. Positive stimulation of the motion area was assumed when movements of the contralateral limb or face were induced, with the concurrent recording of an electromyogram. Positive stimulation affecting the sensation area was assumed when an abnormal feeling was induced in the contralateral limb or face. Positive stimulation of the language area was assumed when the patient exhibited counting interruption, errors during object naming, language confusion or other language problems. During the surgical resection, the patient was asked to repeatedly complete a series of motion and language tasks. If the patient exhibited weakness of physical activity, language abnormalities or abnormal sensation, subcortical DES was performed immediately to confirm the existence of major conduction tracts. The functional areas as determined by cortical or subcortical DES were the areas that the surgery was required not to damage.

Resection. The tumor was resected under the awake status, and the resection was performed to the greatest extent according to the real-time monitoring of changes in language, motion and sensation.

Assessment of postoperative neurological function and extent of resection. At 1 month (early stage) and 3 months (late stage) after the surgery, a detailed assessment of cognitive functions, including nervous system functions and language was performed. The skull was reviewed within $72 \mathrm{~h}$ after the surgery to confirm the extent of tumor resection. For low-grade glioma, T2 or fluid attenuation inversion recovery MRI was used as the reference to determine resection degree, while for high-grade glioma, enhanced T1 was used. Overall resection was $100 \%$ lesion excision; sub-overall resection was $\geq 90 \%$ and $<100 \%$ excision, while partial resection was $<90 \%$ excision.

\section{Results}

Clinical and radiological findings. The patients' general information, clinical manifestations and imaging data are shown in Table I. There were 91 cases of brain function area glioma, including 52 males and 39 females, with an average age of 38.7 years. The clinical manifestations included 73 cases of epilepsy $(80.2 \%)$; 13 cases of headache $(14.3 \%)$ and nine cases of focal neurological dysfunction $(9.9 \%)$. The maximum diameter of the cranial MRI lesions was $2.0-6.0 \mathrm{~cm}$, and all were located around the lateral fissure. The lesions were all located on the left, among which 86 cases were right-handed (94.5\%), three cases were left-handed and two cases were ambidextrous. The MMSE scores of all patients were $\geq 28$ points.

Among the patients, 66 cases were of low-grade glioma (72.5\%), including 43 cases of astrocytoma, 17 cases of oligodendroglioma and six cases of hypo-astrocytoma; and 25 cases 
Table I. Summary of clinical manifestations, imaging data, pathology, intraoperative positioning, resection range and postoperative dysfunction of the 91 glioma cases.

\begin{tabular}{|c|c|}
\hline Variable & Cases $(\%)$ \\
\hline \multicolumn{2}{|l|}{ Gender } \\
\hline Male & $52(57.1)$ \\
\hline Female & $39(42.9)$ \\
\hline \multicolumn{2}{|l|}{ Symptoms and signs } \\
\hline Epilepsy & $73(80.2)$ \\
\hline Headache, increased intracranial pressure & $13(14.3)$ \\
\hline Focal neurological dysfunction & $9(9.9)$ \\
\hline \multicolumn{2}{|l|}{ DES results } \\
\hline Motion cortex & $88(96.7)$ \\
\hline Language cortex, summary & $91(100.0)$ \\
\hline Language cortex, counting interruption & $86(94.5)$ \\
\hline Language cortex, anomia & $21(23.1)$ \\
\hline Language cortex, naming error & $5(5.5)$ \\
\hline \multicolumn{2}{|l|}{ Pathology } \\
\hline Low-grade glioma & $66(72.5)$ \\
\hline High-grade glioma & $25(27.5)$ \\
\hline \multicolumn{2}{|l|}{ Resection degree } \\
\hline Overall resection & $53(58.2)$ \\
\hline Sub-overall resection & $31(34.1)$ \\
\hline Partial resection & $7(7.7)$ \\
\hline \multicolumn{2}{|l|}{ Postoperative neurological dysfunction } \\
\hline No & $42(46.2)$ \\
\hline Early stage language dysfunction & $39(42.9)$ \\
\hline Early motion dysfunction & $27(29.7)$ \\
\hline Permanent neurological dysfunction & $1(1.1)$ \\
\hline
\end{tabular}

were of high-grade glioma (27.5\%), including 15 cases of glioblastoma, five cases of anaplastic astrocytoma and five cases of anaplastic oligodendroglioma.

Cortical sites identified by DES. During the awake surgery, when the stimulus reached a certain intensity (usually 2-4 mA), all the cortical functional areas were stimulated, at which time 88 cases (125 positive stimulation points) exhibited facial or hand movement onset, and 91 cases (112 positive stimulation points) exhibited language disorders or counting interruption, naming error or anomia. The greatest difference among individuals was in the distribution of the points that were determined to be in language areas, although all were concentrated within a range of $1 \mathrm{~cm}^{2}$. Among these, 86 points were associated with counting interruption, 21 points were associated with anomia and 5 points were associated with naming errors. The stimulation points that were positive for counting interruption were mainly located on the posterior part of the left anterior central gyrus (41 points, $47.7 \%$ ), the operculum of the left inferior frontal gyrus (21 points, 24.4\%), the triangular part of the left inferior frontal gyrus (11 points, $12.8 \%$ ), the left posterior middle frontal gyrus (9 points, $10.5 \%)$ and the posterior part of the superior frontal gyrus (4 points, $4.7 \%$; Fig. 1).

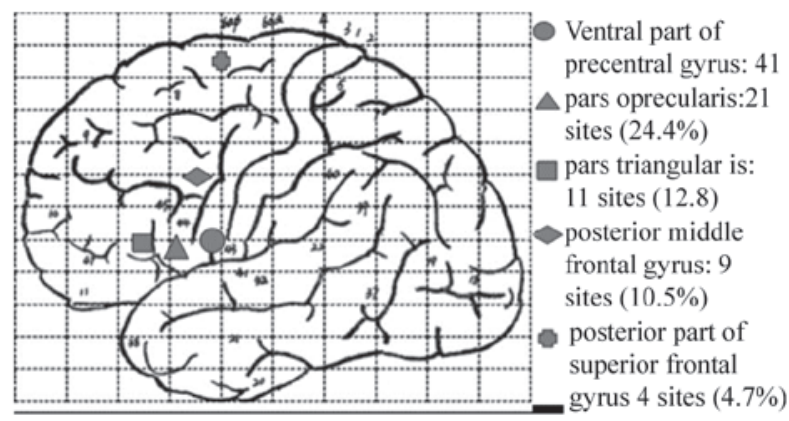

Figure 1. Broca region distribution diagram of counting interruption identified in the direct electrical stimulation of Chinese patients. The sites are mainly located on the posterior part of the left anterior central gyrus $(47.7 \%)$, the operculum of the left inferior frontal gyrus (24.4\%), the triangular part of the left inferior frontal gyrus $(12.8 \%)$, the left posterior middle frontal gyrus $(10.5 \%)$ and the posterior part of the superior frontal gyrus $(4.7 \%)$.

Extent of resection. Cranial MRI was performed $72 \mathrm{~h}$ after the surgery, and demonstrated that overall resection was achieved in 53 cases $(58.2 \%)$, sub-overall resection was performed in 31 cases $(34.1 \%)$ and partial resection was performed in seven cases $(7.7 \%)$.

Intra-operative side-effects. Seven patients exhibited intraoperative electrical stimulation-induced epileptic seizures, which were controlled by the use of ice brine to wash the cortex; A total of 21 patients experienced shivering in the awake procedure prior to the introduction of a heating blanket to the awake surgeries. With regard to discomfort, no patient suffered from unendurable pain, expressed any intraoperative severe pain or had any postoperative pain memory after waking up. Intraoperative discomfort comprised 38 cases of urine-holding (41.8\%), 35 cases of dry mouth (38.5\%) and 10 cases of slight pain in the temporalis muscle or meninges $(11.0 \%)$.

Postoperative neurological status. Postoperatively, 42 cases (46.2\%) had no postoperative neurologic dysfunction; 39 cases (42.9\%) exhibited brief language dysfunction; 27 cases $(29.7 \%)$ exhibited a brief limb motion disorder, which returned to preoperative functional levels within 2 weeks to 1 month after the surgery; and one case (1.1\%) exhibited permanent neurological dysfunction. In that case, postoperative cranial MRI indicated that an infarction was present within the inner vesicle region.

\section{Discussion}

Glioma in the language area is a challenging problem in neurosurgery. The prognosis improves as the amount of glioma removed increases (1-5); however, the patient's language function should be retained postoperatively. As gliomas often involve the language area, the US National Comprehensive Cancer Network glioma surgery guidelines (http://www.nccn. org/professionals/physician_gls/f_guidelines.asp), as well as the Chinese consensus on the diagnosis and treatment of malignant CNS glioma (14), have proposed that the tumor should be maximally safely resected. This means that the tumor volume should be maximally resected, while the language and other important cognitive functions must not be damaged. The key 
of this type of surgery is how to position the language area and lesion range accurately and in real time during the surgery.

Due to differences between individuals and the effects caused by the positioning of brain tumors, functional areas are shifted and remodeled. Thus, the classical anatomical localization of functional areas is likely to have some errors. Although new imaging methods, such as positron emission tomography, fMRI and magnetoencephalography, are able to locate the position of the motion and sensation cortexes, these methods are not very accurate towards the positioning of the language area; for example, the sensitivity of fMRI towards the language area positioning is $59-100 \%$, while the specificity is only $0-97 \%$ (4). These methods are not able to achieve intraoperative real-time monitoring of the brain functional areas, and cannot perform the positioning of cerebral white matter fibers. Although they are able to identify the cortical area that is associated with a certain function, they are not able to determine which parts are the necessary parts (15). Currently, the most accurate and reliable method of brain functional area positioning is intraoperative cortical or subcortical DES. In real time, DES can determine the parts essential for motion, sensation, language, and even memory, and is able to identify intraoperatively the positions of functional areas located towards the cortex and subcortex of the cerebrum, brainstem and spinal cord $(16,17)$.

In 1870, Fritsch and Hitzig first used this method to locate the brain areas responsible for motion in a dog (18). In 1874, Bartholow, an American neurological surgeon, first performed electric stimulation of a patient's brain, and recorded the associated motion response (19). Penfield and Flanigin (20), a Canadian neurosurgeon, maturely applied this technology, and described in detail the cerebral positioning areas of language, vision and audition, and based on these applications, the famous cortical homunculus map was established. Subsequently, DES spread rapidly throughout Western countries. Berger et al (21) and Duffau (22) applied DES to brain tumor surgeries, particularly low-grade glioma surgeries and concurrently carried out a large number of clinical studies in which the DES technology was applied to the location of subcortical pathways. All the aforementioned efforts have contributed outstandingly to the promotion of DES, making it one of the essential techniques in the functional area surgery of neurosurgery (23-26). In 2002, Wang (Guangzhou, China) applied DES to the surgery of brain functional area lesions, and following four periods of nationwide study and training, this technology has gradually been accepted and promoted by neurosurgeons in China $(11,12)$.

DES is a safe and reliable positioning method; histological examination has revealed no inflammation or other injuries in the stimulated parts, and patient follow-up has also revealed no significant complications. However, if the stimulation method is not conducted correctly, it would be likely to cause false positive or false negative results, or even status epilepticus. This would affect the surgery, and further cause postoperative permanent neurological dysfunction. Therefore, it is particularly important for the correct stimulation method and stimulation parameters to be used in intraoperative DES (27). The authors of the present study use a biphasic square wave, as a sine wave would cause adaptive adjustment of the cell membranes during the stimulation process, thus increasing the required stimulus current and potentially causing false positive results or the induction of epileptic seizures. The use of a biphasic square wave could avoid the overlaying of current around the cell membranes and the subsequent ionization hydrolysis and heating of local cerebrospinal fluid particles, which would be likely to result in nerve cell damage (7). The stimulation frequency was $60 \mathrm{~Hz}$, and the duration of the stimulating square-wave was $1 \mathrm{msec}$. The current was determined based on the appearance of post-discharge stimulus during electroencephalography monitoring. The stimulation intensity was usually set as a level lower than the lowest current level that induced a post potential; for example, if the current that induced a post potential was $5 \mathrm{~mA}$, the stimulus current was set at $4 \mathrm{~mA}$. The stimulus current was normally applied at $1 \mathrm{~mA}$ initially, and increased in increments of $1 \mathrm{~mA}$, usually reaching 2-4 $\mathrm{mA}$. The stimulus duration for the motion and sensation tasks was $\sim 1 \mathrm{sec}$, and that for the language and cognitive tasks was $\sim 4 \mathrm{sec}$ (28). It has been observed in certain studies, particularly that regarding patients with epilepsy, the square wave duration used to stimulate the positioned functional areas was $0.2 \mathrm{msec}$; therefore, the stimulus current was significantly greater than that used in the present study (29). In summary, to prevent the induction of status epilepticus it is recommended to avoid a high stimulus frequency, long stimulus duration, excessive stimulus current, post discharge; and two consecutive positive stimuli.

DES is a reliable and noninvasive method for cerebral functional area positioning, and provides a new surgical concept for glioma resection in the language area, which promotes the modeling of the language area during glioma surgery by a functional method rather than an anatomical one. The operative range using DES reaches the functional border rather than the anatomical border; thus, peritumoral tissues, which may be invaded by the tumors, may also be resected in certain cases. For low-grade glioma, the tumor may be resected to the maximum extent under the premise of retaining the important brain functions, thus prolonging the survival period. For high-grade glioma, cortical DES avoids surgery-induced functional deficits, and improve the patients' survival qualities (30-33). De Witt Hamer et al (9) completed a meta-analysis, which collected 90 documents and the surgical situations of 8,091 glioma cases, and found that the rate of long-term severe neurological dysfunction subsequent to DES was $3.4 \%$, while the long-term severe disability rate of patients that underwent surgery without DES was $8.2 \%$. Furthermore, for the patients undergoing DES, the overall resection rate and the rate of involvement of the language functional area in the resection were significantly increased. The present study also demonstrated that under the premise of not reducing the resection extent, although the incidence of early neurological disorder was high (53.8\%), the incidence of long-term neurological dysfunction was also very low $(1.1 \%)$, indicating that the aim of maximum safe resection could be realized.

Furthermore, DES also provides an important research method for neurosurgeons to understand the higher cognitive functions of the brain. DES plays an important role in studies of such higher cognitive functions as hemispheric ignorance, perceptual awareness, music, calculation, memory and category-specific naming (12,34-39). In the present study, it was found that the Broca area of a Chinese population undergoing DES was mainly concentrated within a $1-\mathrm{cm}^{2}$ 
range. However, great differences existed among individuals. The zones that tested positive for counting interruption were mainly located on the posterior part of the left anterior central gyrus $(47.7 \%)$, the operculum of the left inferior frontal gyrus $(24.4 \%)$, the triangular part of the left inferior frontal gyrus (12.8\%), the left posterior middle frontal gyrus (10.5\%) and the posterior part of the superior frontal gyrus (4.7\%).

In summary, cortical DES was found to be a reliable non-invasive method for cerebral functional area positioning. The application of this technology in glioma surgery of the language area may achieve the maximally safe resection of tumors, and provide assistance towards the cortical positioning of cerebral functional areas in the Chinese population.

\section{References}

1. Chan-Seng E, Moritz-Gasser S and Duffau H: Awake mapping for low-grade gliomas involving the left sagittal stratum: Anatomofunctional and surgical considerations. J Neurosurg 120: 1069-1077, 2014.

2. Sanai $\mathrm{N}$ and Berger MS: Glioma extent of resection and its impact on patient outcome. Neurosurgery 62: 753-764, 2008.

3. Han SJ and Sughrue ME: The rise and fall of 'biopsy and radiate': A history of surgical nihilism in glioma treatment. Neurosurg Clin N Am 23: 207-214, 2012.

4. Giussani C, Roux FE, Ojemann J, Sganzerla EP, Pirillo D and Papagno C: Is preoperative functional magnetic resonance imaging reliable for language areas mapping in brain tumor surgery? Review of language functional magnetic resonance imaging and direct cortical stimulation correlation studies. Neurosurgery 66: 113-120, 2010.

5. Choi BD, Mehta AI, Batich KA, Friedman AH and Sampson JH: The use of motor mapping to aid resection of eloquent gliomas. Neurosurg Clin N Am 23: 215-225, 2012.

6. Lubrano V, Draper L and Roux FE: What makes surgical tumor resection feasible in Broca's area? Insights into intraoperative brain mapping. Neurosurgery 66: 868-875, 2010

7. Mandonnet E, Winkler PA and Duffau H: Direct electrical stimulation as an input gate into brain functional networks: Principles, advantages and limitations. Acta Neurochir (Wien) 152: 185-193, 2010.

8. Duffau H: Awake surgery for nonlanguage mapping. Neurosurgery 66: 523-528, 2010.

9. De Witt Hamer PC, Robles SG, Zwinderman AH, Duffau H and Berger MS: Impact of intraoperative stimulation brain mapping on glioma surgery outcome: a meta-analysis. J Clin Oncol 30: 2559-2565, 2012

10. Tan LH, Spinks JA, Eden GF, Perfetti CA and Siok WT: Reading depends on writing, in Chinese. Proc Natl Acad Sci USA 102: 8781-8785, 2005.

11. Bai HM, Wang WM, Li TD, et al: Three core techniques in surgery of neuroepithelial tumors in eloquent areas: awake anaesthesia, intra-operative direct electrical stimulation and ultrasonography. Chin Med J (Engl) 124: 3035-3041, 2011.

12. Bai HM, Jiang T, Wang WM, Li TD, Liu Y and Lu YC: Functional MRI mapping of category-specific sites associated with naming of famous faces, animals and man-made objects. Neurosci Bull 27: 307-318, 2011.

13. Oldfield RC: The assessment and analysis of handedness: The Edinburgh inventory. Neuropsychologia 9: 97-113, 1971

14. Zhou LF, Wang RZ, Bao SD et al: Chinese guideline for diagnosis and treatment on central nervous system tumors. Zhonghua $\mathrm{Yi}$ Xue Za Zhi 92: 2309-2313, 2012 (In Chinese).

15. Kim SS, McCutcheon IE, Suki D, et al: Awake craniotomy for brain tumors near eloquent cortex: correlation of intraoperative cortical mapping with neurological outcomes in 309 consecutive patients. Neurosurgery 64: 836-845, 2009.
16. Sacko O, Lauwers-Cances V, Brauge D, Sesay M, Brenner A and Roux FE: Awake craniotomy vs surgery under general anesthesia for resection of supratentorial lesions. Neurosurgery 68: 1192-1198, 2011

17. De Benedictis A, Moritz-Gasser S and Duffau H: Awake mapping optimizes the extent of resection for low-grade gliomas in eloquent areas. Neurosurgery 66: 1074-1084, 2010.

18. Fritsch G and Hitzig E: Electric excitability of the cerebrum (Uber die elektrische Erregbarkeit des Grosshirns). Epilepsy Behav 15: 123-130, 2009

19. Bartholow R: Experimental investigations into the functions of the human brain. Am J Med Sci 66: 305-313, 1874.

20. Penfield W and Flanigin H: Surgical therapy of temporal lobe seizures. AMA Arch Neurol Psychiatry 64: 491-500, 1950.

21. Berger MS, Stieg PE, Danks RA, Schwartz RB and Folkerth RD: Lesions in eloquent cortex. Neurosurgery 40: 1059-1063, 1997.

22. Duffau H: Surgery of low-grade gliomas: Towards a 'functional neurooncology'. Curr Opin Oncol 21: 543-549, 2009.

23. Ilmberger J, Ruge M, Kreth FW, Briegel J, Reulen HJ and Tonn JC: Intraoperative mapping of language functions: A longitudinal neurolinguistic analysis. J Neurosurg 109: 583-592, 2008.

24. Desmurget M, Reilly KT, Richard N, Szathmari A, Mottolese C and Sirigu A: Movement intention after parietal cortex stimulation in humans. Science 324: 811-813, 2009.

25. Roux FE, Dufor O, Lauwers-Cances V, et al: Electrostimulation mapping of spatial neglect. Neurosurgery 69: 1218-1231, 2011.

26. Beez T, Boge K, Wager M, et al; European Low Grade Glioma Network: Tolerance of awake surgery for glioma: A prospective European Low Grade Glioma Network multicenter study. Acta Neurochir (Wien) 155: 1301-1308, 2013

27. De Benedictis A, Sarubbo S and Duffau H: Subcortical surgical anatomy of the lateral frontal region: Human white matter dissection and correlations with functional insights provided by intraoperative direct brain stimulation: laboratory investigation. J Neurosurg 117: 1053-1069, 2012.

28. Duffau H: A new concept of diffuse (low-grade) glioma surgery. Adv Tech Stand Neurosurg 38: 3-27, 2012.

29. Lüders HO: Symptomatic Areas and Electrical Cortical Stimulation. New York: Churchill Livingstone, 2000.

30. Duffau H: Awake surgery for incidental WHO grade II gliomas involving eloquent areas. Acta Neurochir (Wien) 154: 575-584, 2012.

31. Duffau H: Brain mapping in tumors: Intraoperative or extraoperative? Epilepsia 54: 79-83, 2013.

32. Duffau H: A new philosophy in surgery for diffuse low-grade glioma (DLGG): Oncological and functional outcomes. Neurochirurgie 59: 2-8, 2013.

33. Duffau $\mathrm{H}$ and Mandonnet E: The 'onco-functional balance' in surgery for diffuse low-grade glioma: Integrating the extent of resection with quality of life. Acta Neurochir (Wien) 155: 951-957, 2013.

34. Gras-Combe G, Moritz-Gasser S, Herbet G and Duffau H: Intraoperative subcortical electrical mapping of optic radiations in awake surgery for glioma involving visual pathways. J Neurosurg 117: 466-473, 2012.

35. Klein M, Duffau H and De Witt Hamer PC: Cognition and resective surgery for diffuse infiltrative glioma: An overview. J Neurooncol 108: 309-318, 2012.

36. Maldonado IL, Moritz-Gasser S, de Champfleur NM, Bertram L, Moulinié G and Duffau H: Surgery for gliomas involving the left inferior parietal lobule: new insights into the functional anatomy provided by stimulation mapping in awake patients. J Neurosurg 115: 770-779, 2011.

37. Pallud J, Mandonnet E and Duffau H: Diffuse low-grade gliomas and UCSF scores. J Neurosurg 120: 577-578, 2014.

38. Schucht P, Ghareeb F and Duffau H: Surgery for low-grade glioma infiltrating the central cerebral region: location as a predictive factor for neurological deficit, epileptological outcome and quality of life. J Neurosurg 119: 318-323, 2013.

39. Yordanova YN, Moritz-Gasser S and Duffau H: Awake surgery for WHO Grade II gliomas within 'noneloquent' areas in the left dominant hemisphere: toward a 'supratotal' resection. Clinical article. J Neurosurg 115: 232-239, 2011. 